Supporting Information

$\mathrm{NiCo}_{2} \mathrm{~S}_{4}$ nanotubes anchored 3D nitrogen-doped graphene framework as electrode material with enhanced performance for asymmetric supercapacitors

Yiying Chen a, Tao Liu ${ }^{\text {a }}$, Liuyang Zhang a, ${ }^{\text {* }}$ and Jiaguo $\mathrm{Yu}^{\text {a, b, * }}$

Page: 18

Figures: 17

Tables: 2 


\section{Materials characterization}

Powder X-ray diffraction (XRD) patterns was obtained on a Rigaku X-ray diffractometer with $\mathrm{Cu} \mathrm{K \alpha}$ irradiation $(\lambda=0.15418 \mathrm{~nm})$. Microstructure images were attained from field-emission scanning electron microscopy (FESEM, JSM-7500) and transmission electron microscopy (TEM, Titan G2). Measurements of the BrunauerEmmett-Teller $(\mathrm{BET})$ specific surface area $\left(S_{\mathrm{BET}}\right)$ were carried out at Micromeritics ASAP 3020 nitrogen adsorption apparatus and the pore size distribution was determined via the Barret-Joyner-Halender $(\mathrm{BJH})$ method. The X-ray photoelectron spectroscopy (XPS) was performed on Thermo ESCALA 250. Raman spectrum analysis was conducted by a Renishaw Raman microscope under $\lambda_{\mathrm{exc}}=633 \mathrm{~nm}$ laser excitation. Contact angle was tested by using the Theta instrument (Biolin Scientific). Atomic force microscopy (AFM) was employed bySPM-9700HT (Shimadzu, Japan).

\section{Electrochemical measurements}

The working electrodes were fabricated by brushing a mixture onto a piece of cleaned nickel foam and then air-dried at $100^{\circ} \mathrm{C}$ overnight. The mixture was prepared by mixing the as-prepared samples with acetylene black and polyvinylidene fluoride at a mass ratio of 8:1:1 in N-Methyl pyrrolidone (NMP) solvent. The mass loading of active material was about $1.0 \mathrm{mg} \mathrm{cm}^{-2}$. Electrochemical performance of the electrodes were conducted in a three-electrode configuration in $2 \mathrm{M} \mathrm{KOH}$ using $\mathrm{CHI} 760 \mathrm{E}$ electrochemical workstation, where platinum foil and $\mathrm{Ag} / \mathrm{AgCl}$ electrode were served as counter electrode and reference electrode, respectively. Cyclic voltammetry (CV), 
galvanostatic charge/discharge (GCD) and electrochemical impedance spectroscopy (EIS) were tested on electrochemical workstation (CHI 760E). Cycle life was performed on a battery test system (LANHE CT2001A).

In a typical three-electrode system, the specific capacitance and capacity of the working electrode can be obtained from the equation:

$$
\begin{aligned}
& C=\frac{I \times \Delta t}{m \times \Delta V} \\
& C S=\frac{I \times \Delta t}{m}
\end{aligned}
$$

Where $C\left(\mathrm{~F} \mathrm{~g} \mathrm{~g}^{-1}\right)$ is the specific capacitance, $C s\left(\mathrm{C} \mathrm{g}^{-1}\right)$ is the specific capacity, $I$ (A) is the charging-discharging current, $\Delta V(\mathrm{~V})$ is the potential window, $\Delta t(\mathrm{~s})$ is the discharging time, and $m(\mathrm{~g})$ is the loading mass of active material only.

\section{Fabrication and evaluation of asymmetric supercapacitors (ASCs)}

ASCs were fabricated by assembling NCS/NGF or NCS as positive electrode, active carbon as negative electrode and $2 \mathrm{M} \mathrm{KOH}$ aqueous electrolyte with a piece of glassy fibrous paper as separator in a 2025 type coin cell.

In order to acquire the optimum electrochemical performance of the asymmetric supercapacitors, charge balance principle is used to determine the mass ratio of the positive to negative electrode which can be calculated by the following equation:

$$
\frac{m_{-}}{m_{+}}=\frac{C_{-} \times \Delta V_{-}}{C_{+} \times \Delta V_{+}}
$$

where $m_{-}$is the loading mass of cathode active-material and $m_{+}$is the loading mass of anode active-material, $C_{+}$and $C_{-}$are the specific capacitance of the NCS/NGF electrodes and $\mathrm{AC}$ electrodes, respectively, and $\Delta V_{+}$and $\Delta V_{-}$are the potential 
range.

CV and GCD tests were tested on CHI 760E electrochemical workstation. Cycle life measurement was tested on LANHE CT2001A. The specific capacitance (C), energy density (E) and power density of ASC devices are evaluated based on the following equations:

$$
\begin{gathered}
C=\frac{I \times \Delta t}{M \times \Delta V} \\
E=\frac{1}{3.6} \times \frac{1}{2} \times C \times V^{2} \\
P=3600 \times \frac{E}{\Delta t}
\end{gathered}
$$

Where $C\left(\mathrm{~F} \mathrm{~g} \mathrm{~g}^{-1}\right)$ is the specific capacitance of the ASC cell, $M(\mathrm{~g})$ is the total mass of active materials of anode and cathode, $V(\mathrm{~V})$ is the voltage window and $\Delta t(\mathrm{~s})$ is the discharging time during the GCD measurement.
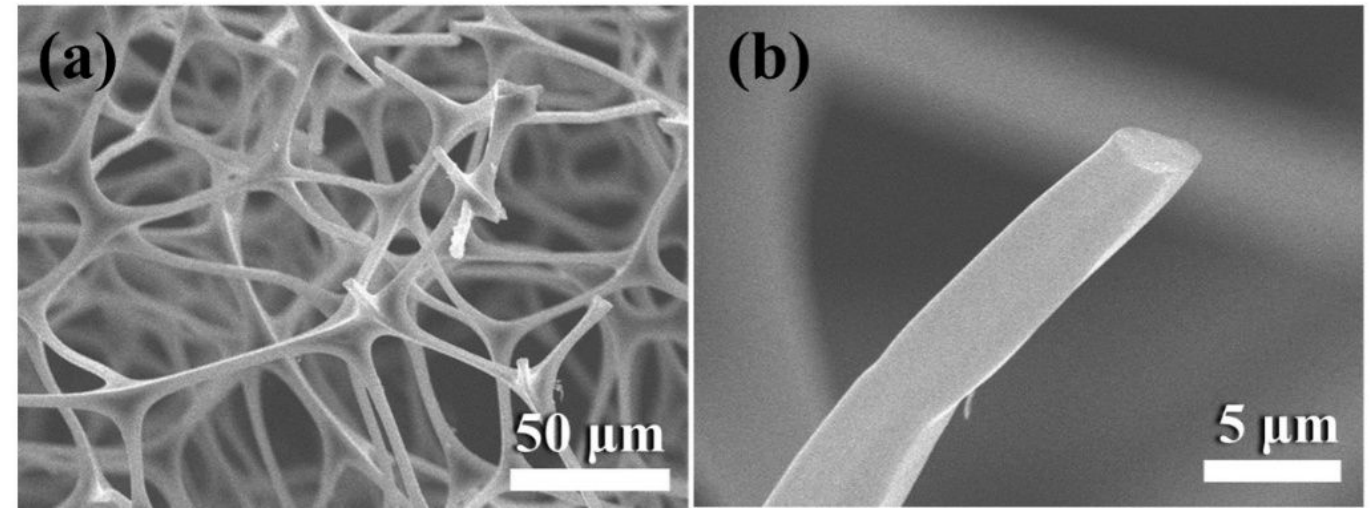

Figure S1. SEM images of melamine sponge with different magnifications. 

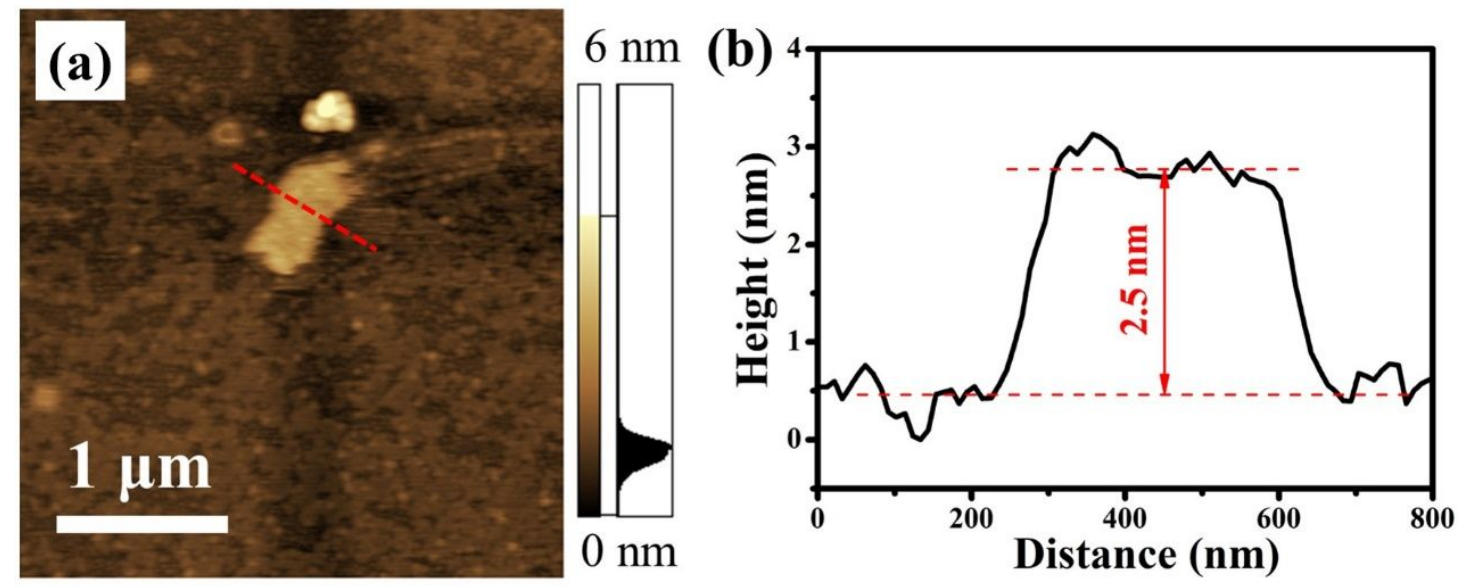

Figure S2. (a) AFM image and (b) the corresponding height profile of NGF fragment .

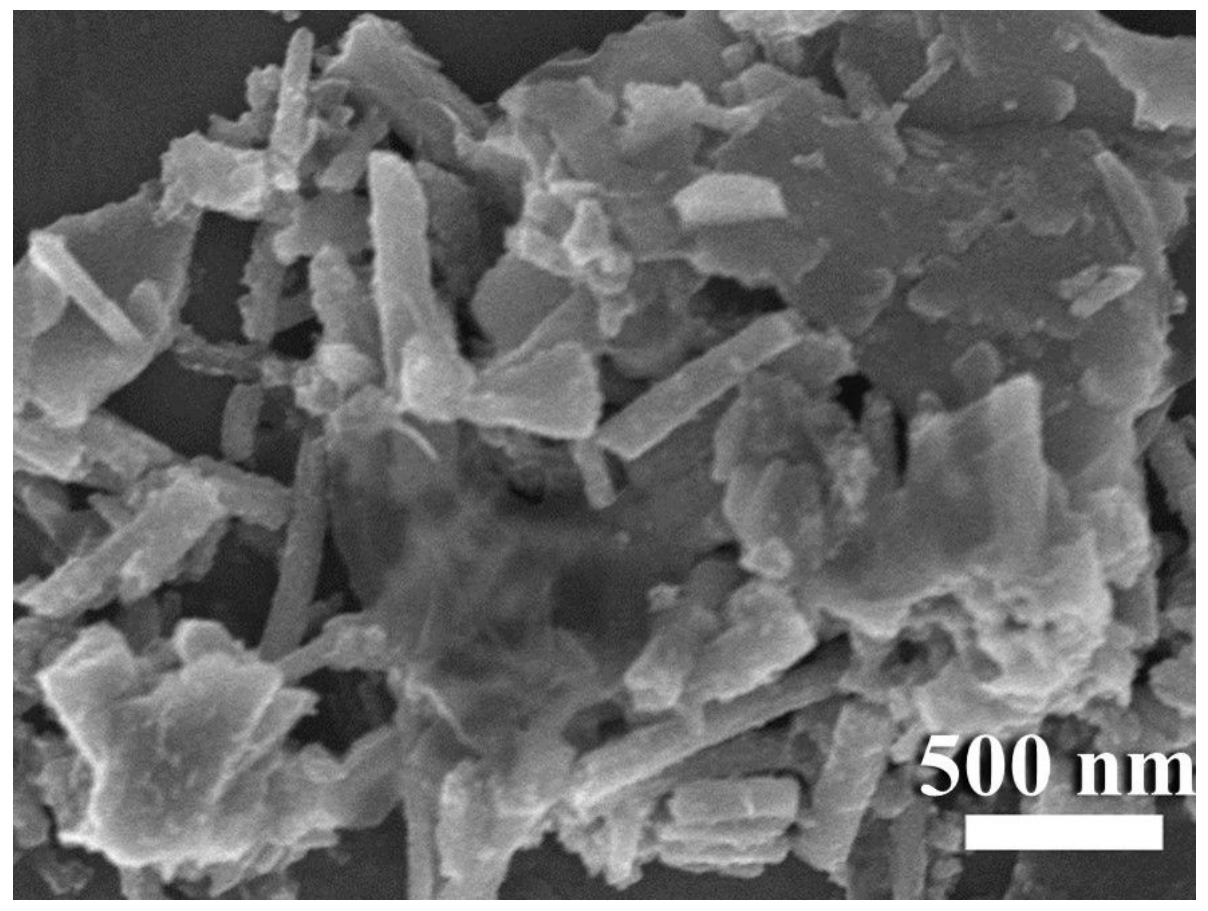

Figure S3. FESEM image of the GO/NCS composite. 

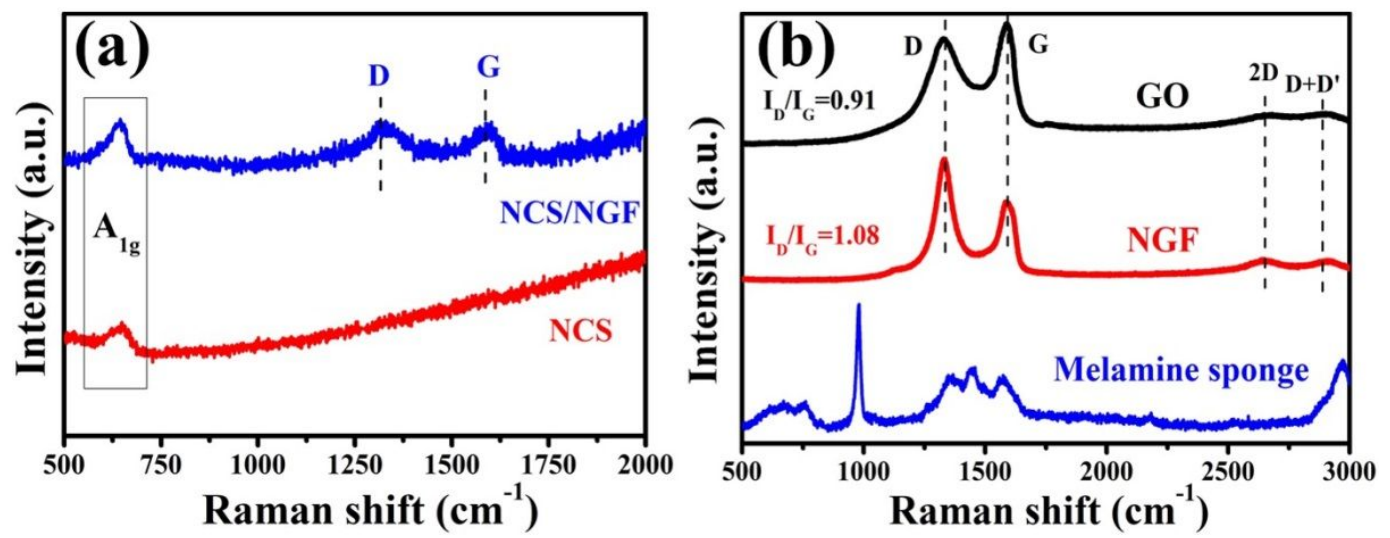

Figure S4. Raman spectra of (a) GO, NGF, NCS/NGF and NCS; (b) GO and NGF with larger measure range.

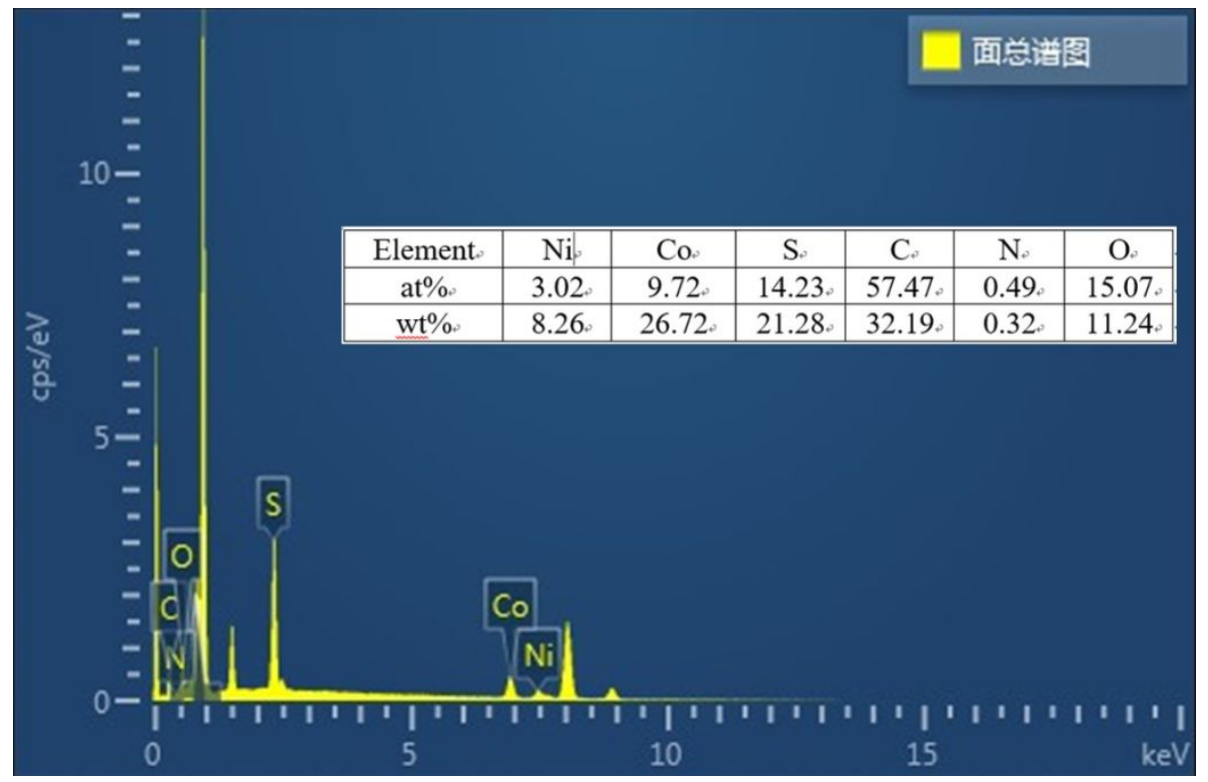

Figure S5. EDX spectrum of the NCS/NGF composite 

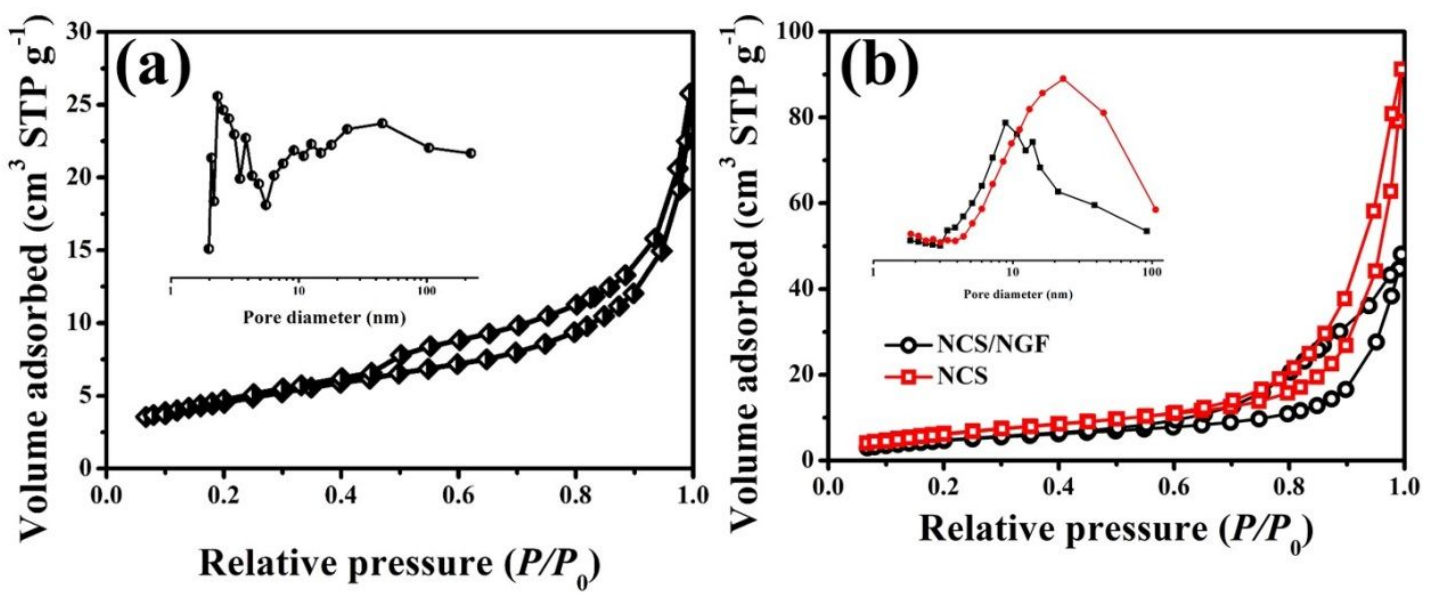

Figure S6. $\mathrm{N}_{2}$ adsorption/desorption isotherm and the corresponding pore size distribution (inset) of NGF (a) and bare NCS and NCS/NGF nanocomposite (b)

Table S1. Pore textural properties of NCS, NCS/NGF and NGF.

\begin{tabular}{cccc}
\hline Samples & $S_{\text {BET }}\left(\mathrm{m}^{2} \mathrm{~g}^{-1}\right)$ & $V_{\text {pore }}\left(\mathrm{m}^{3} \mathrm{~g}^{-1}\right)$ & $D_{\text {pore }}(\mathrm{nm})$ \\
\hline NCS & 25 & 0.14 & 22.5 \\
NCS/NGF & 19 & 0.07 & 16.1 \\
NGF & 16 & 0.04 & 9.7 \\
\hline
\end{tabular}

$S_{\mathrm{BET}}$ : BET surface area; $V_{\text {pore: }}$ total pore volume (less than $370 \mathrm{~nm}$ at $P / P_{0}=0.995$ ); $D_{\text {pore: }}$ : average pore diameter.
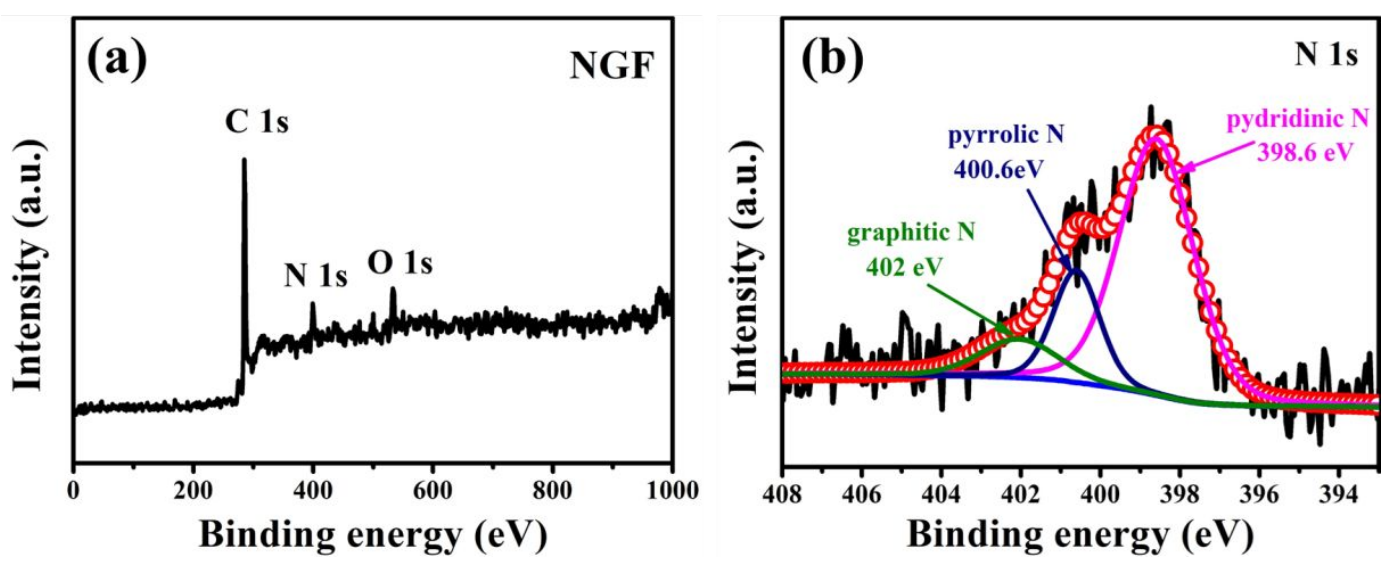

Figure S7. (a) XPS survey scan and high resolution XPS spectrum of (b) N 1s spin- 
orbits.

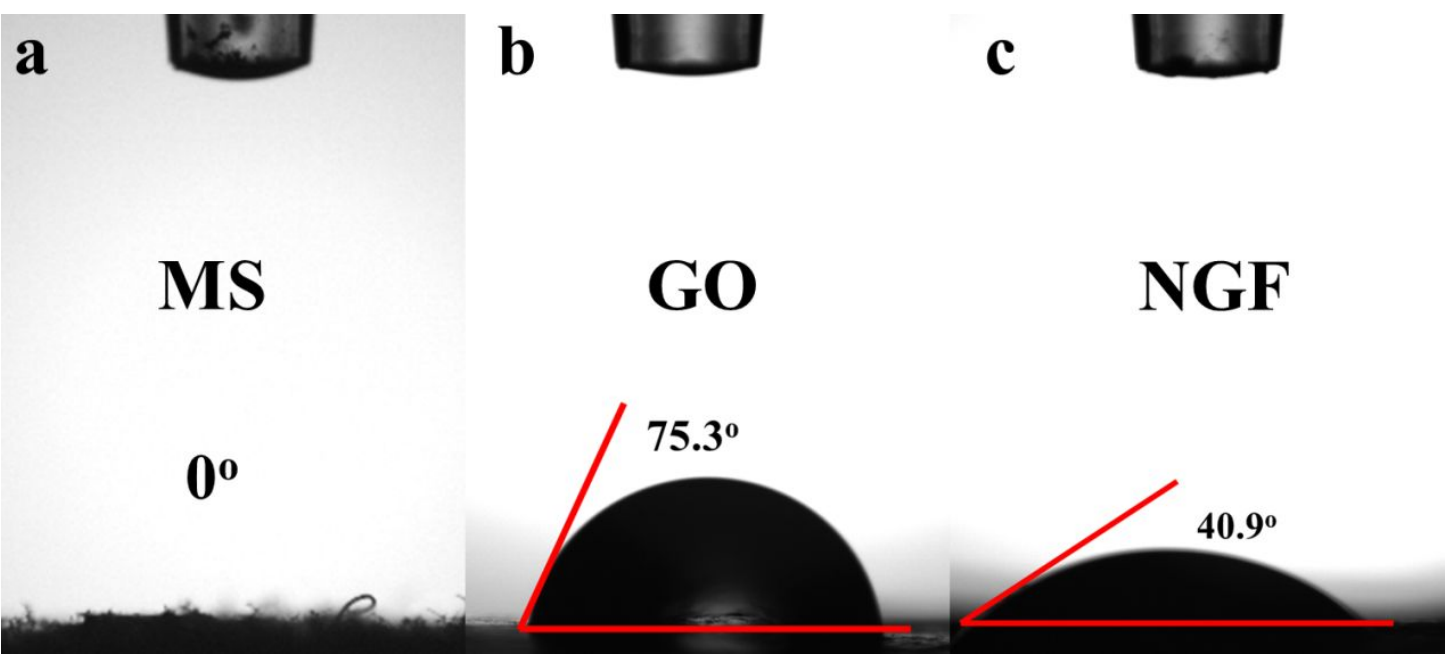

Figure S8. Contact angles of de-ionized (DI) water for (a) melamine sponge (MS), (b)

GO and (c) NGF
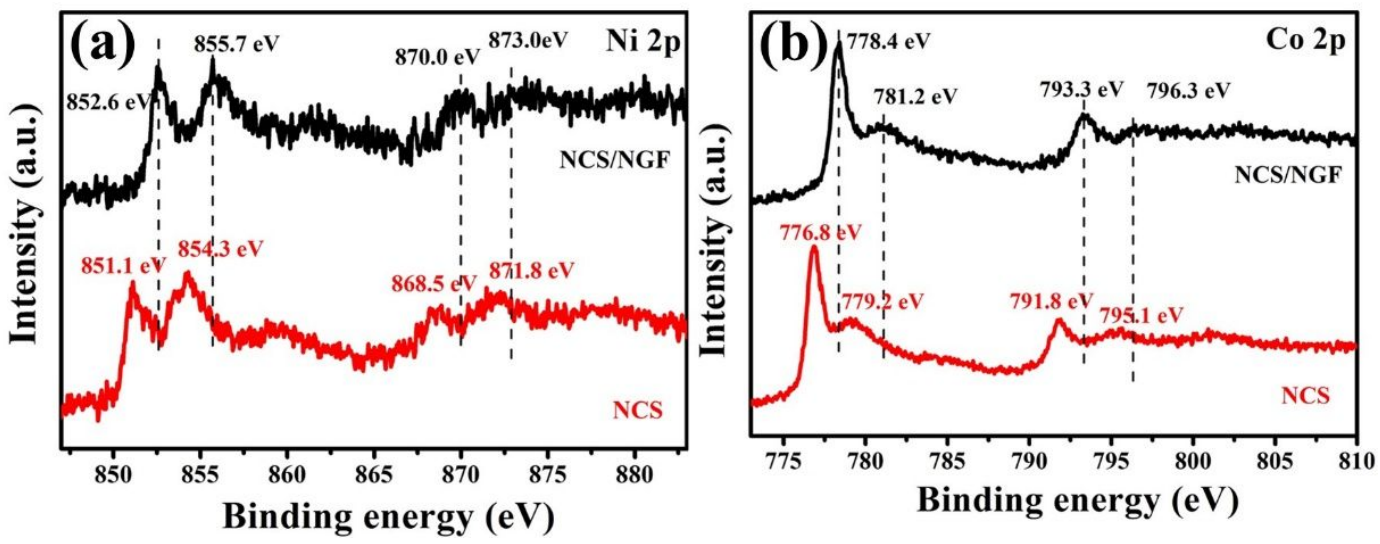

Figure S9. Comparison of (a) Ni $2 p$ spectra and (b) Co $2 p$ spretra of NCS/NGF and NCS. 

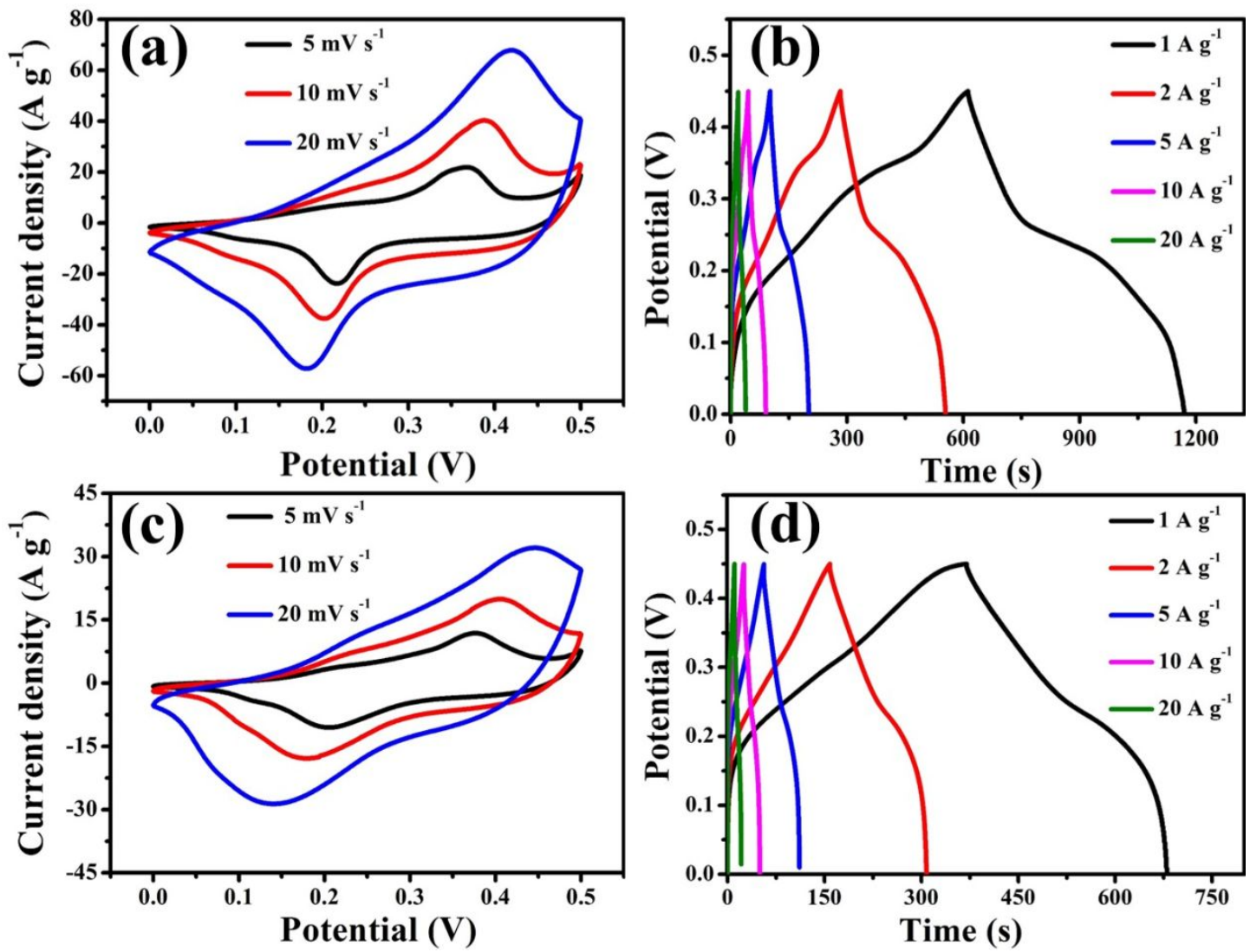

Figure S10. (a) CV curves and (b) GCD curves of NCS/NGF electrode. (c) CV curves and (d) GCD curves of NCS electrode.
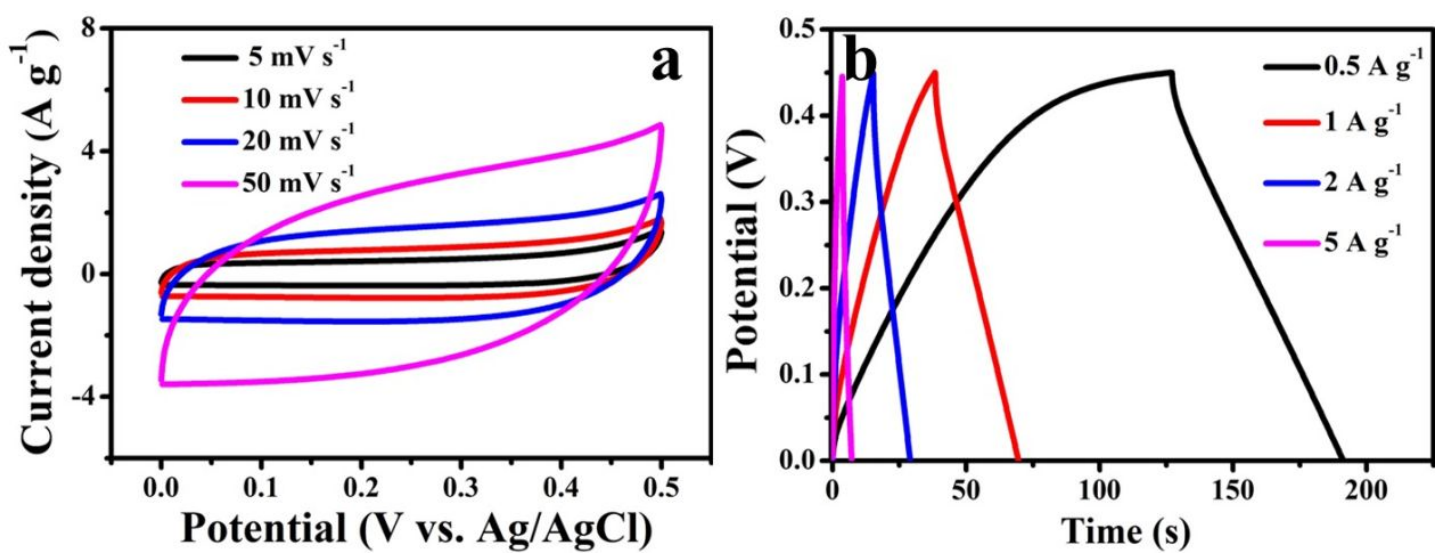

Figure S11. (a) CV curves and (b) GCD curves of NGF.

Table S2. Capacitive performance of different Ni-Co related electrode. 


\begin{tabular}{|c|c|c|c|c|}
\hline Electrode material & $\begin{array}{l}\text { Specific } \\
\text { capacitance } \\
\left(\mathrm{F} \mathrm{g}^{-1}\right)\end{array}$ & $\begin{array}{l}\text { Current } \\
\text { density } \\
\left(\mathrm{A} \mathrm{g}^{-1}\right)\end{array}$ & Capacitance retention & Ref. \\
\hline $\mathrm{NiCo}_{2} \mathrm{~S}_{4} / \mathrm{NGF}$ & 1240 & 1 & $\begin{array}{l}80 \% \text { after } 5000 \text { cycles } \\
\text { at } 10 \mathrm{~A} \mathrm{~g}^{-1}\end{array}$ & $\begin{array}{l}\text { This } \\
\text { work }\end{array}$ \\
\hline $\begin{array}{l}\text { Porous } \\
\mathrm{NiCo}_{2} \mathrm{~S}_{4} / \mathrm{rGO}(16 \%)\end{array}$ & 1107 & 1 & $\begin{array}{l}90 \% \text { after } 5000 \text { cycles } \\
\text { at } 5 \mathrm{~A} \mathrm{~g}^{-1}\end{array}$ & 1 \\
\hline $\begin{array}{l}\text { Hollow } \\
\mathrm{NiCo}_{2} \mathrm{~S}_{4} / \mathrm{Co}_{9} \mathrm{~S}_{8} \\
\text { spindles }\end{array}$ & 749 & 4 & $\begin{array}{l}78 \% \text { after } 5000 \text { cycles } \\
\text { at } 10 \mathrm{~A} \mathrm{~g}^{-1}\end{array}$ & 2 \\
\hline $\begin{array}{l}\mathrm{NiCo}_{2} \mathrm{~S}_{4} \text { porous } \\
\text { nanotubes }\end{array}$ & 933 & 1 & $\begin{array}{l}63 \% \text { after } 5000 \text { cycles } \\
\text { at } 1 \mathrm{~A} \mathrm{~g}^{-1}\end{array}$ & 3 \\
\hline $\mathrm{NiCo}_{2} \mathrm{~S}_{4}$ nanosheets & 744 & 1 & $\begin{array}{l}93.4 \% \text { after } 1500 \\
\text { cycles at } 2 \mathrm{~A} \mathrm{~g}^{-1}\end{array}$ & 4 \\
\hline $\begin{array}{l}\mathrm{NiCo}_{2} \mathrm{~S}_{4} \\
\text { nanoparticles/PRGO }\end{array}$ & 1090 & 2 & $\begin{array}{l}80.1 \% \text { after } 3000 \\
\text { cycles at } 10 \mathrm{~A} \mathrm{~g}^{-1}\end{array}$ & 5 \\
\hline $\begin{array}{l}\mathrm{NiCo}_{2} \mathrm{~S}_{4} \\
\text { nanoparticles }\end{array}$ & 972 & 2 & $\begin{array}{l}107.9 \% \text { after } 1000 \\
\text { cycles at } 3 \mathrm{~A} \mathrm{~g}^{-1}\end{array}$ & 6 \\
\hline $\begin{array}{l}\mathrm{NiCo}_{2} \mathrm{~S}_{4} \text { nanoflakes } \\
\text { on Ppy nanotubes }\end{array}$ & 908.1 & 1 & $\begin{array}{l}87.7 \% \text { after } 2000 \\
\text { cycles at } 20 \mathrm{~A} \mathrm{~g}^{-1}\end{array}$ & 7 \\
\hline $\begin{array}{l}\mathrm{NiCo}_{2} \mathrm{~S}_{4} \text { urchin-like } \\
\text { nanostructures }\end{array}$ & 1149 & 1 & $\begin{array}{l}91.4 \% \text { after } 5000 \\
\text { cycles at } 20 \mathrm{~A} \mathrm{~g}^{-1}\end{array}$ & 8 \\
\hline $\begin{array}{l}\text { Onion-Like } \mathrm{NiCo}_{2} \mathrm{~S}_{4} \\
\text { particles }\end{array}$ & 1016 & 2 & $\begin{array}{l}87 \% \text { after } 10000 \\
\text { cycles at } 10 \mathrm{~A} \mathrm{~g}^{-1}\end{array}$ & 9 \\
\hline $\begin{array}{l}\text { Hollow hexagonal } \\
\mathrm{NiCo}_{2} \mathrm{~S}_{4} \text { nanoplates }\end{array}$ & 437 & 1 & $\begin{array}{l}81 \% \text { after } 1000 \text { cycles } \\
\text { at } 2 \mathrm{~A} \mathrm{~g}^{-1}\end{array}$ & 10 \\
\hline $\begin{array}{l}3 \mathrm{D} \mathrm{Ni} \mathrm{Co}_{1-\mathrm{X}} \mathrm{S}_{2} \\
\text { particles/graphene }\end{array}$ & 1166 & 1 & $\begin{array}{l}74.5 \% \text { after } 1000 \\
\text { cycles at } 5 \mathrm{~A} \mathrm{~g}^{-1}\end{array}$ & 11 \\
\hline
\end{tabular}




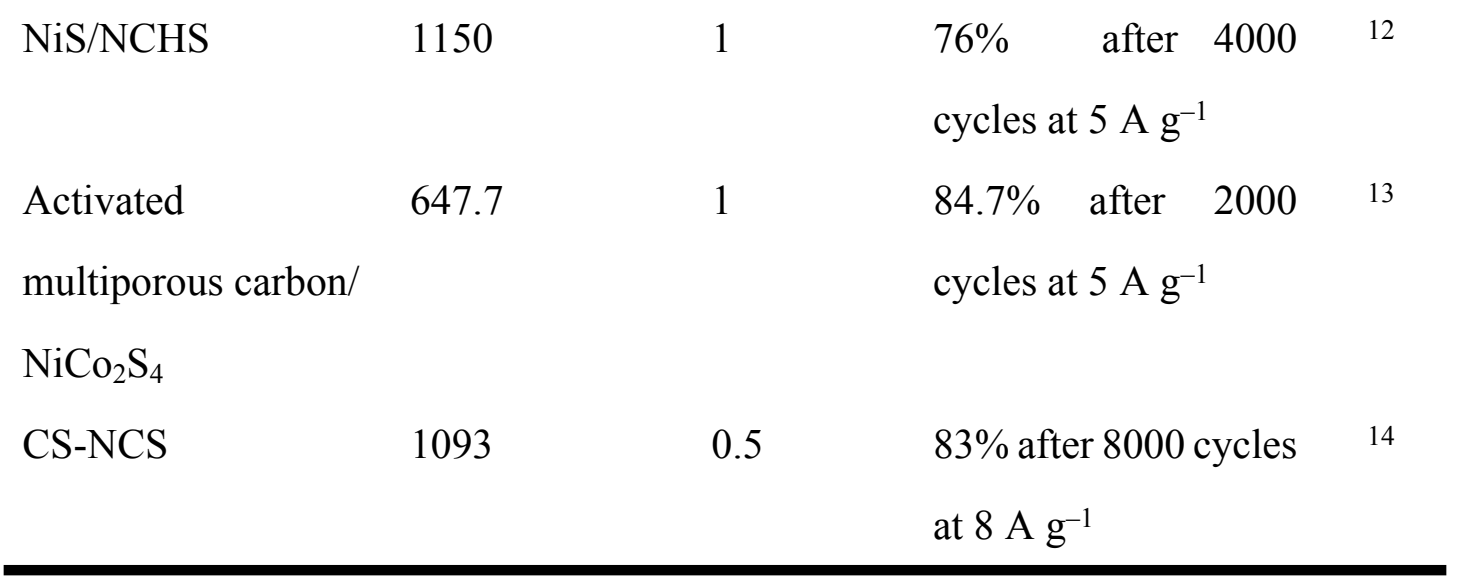



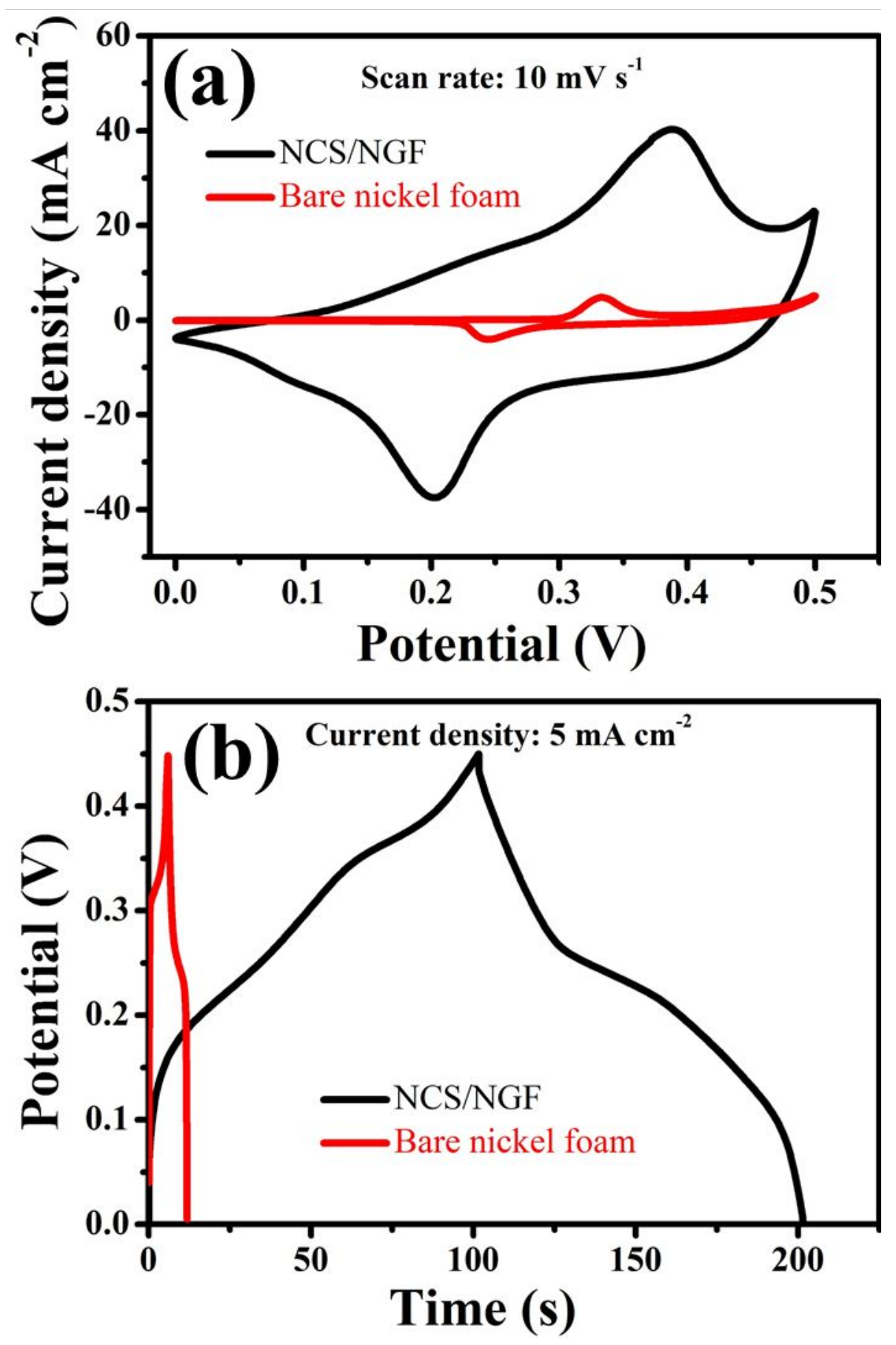

Figure S12. CV and GCD curves for bare nickel foam and NCS/NGF electrodes. 


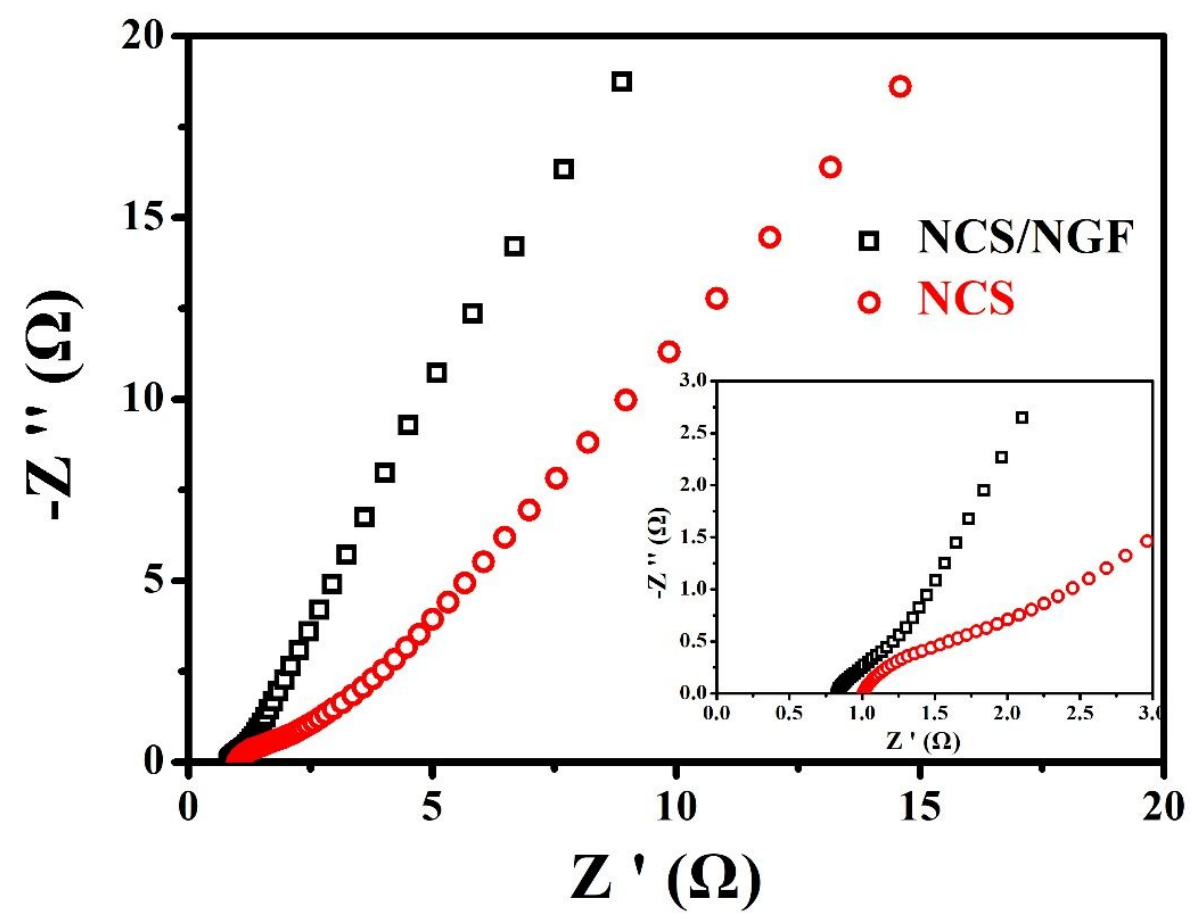

Figure S13. Nyquist plots of NCS/NGF and bare NCS.
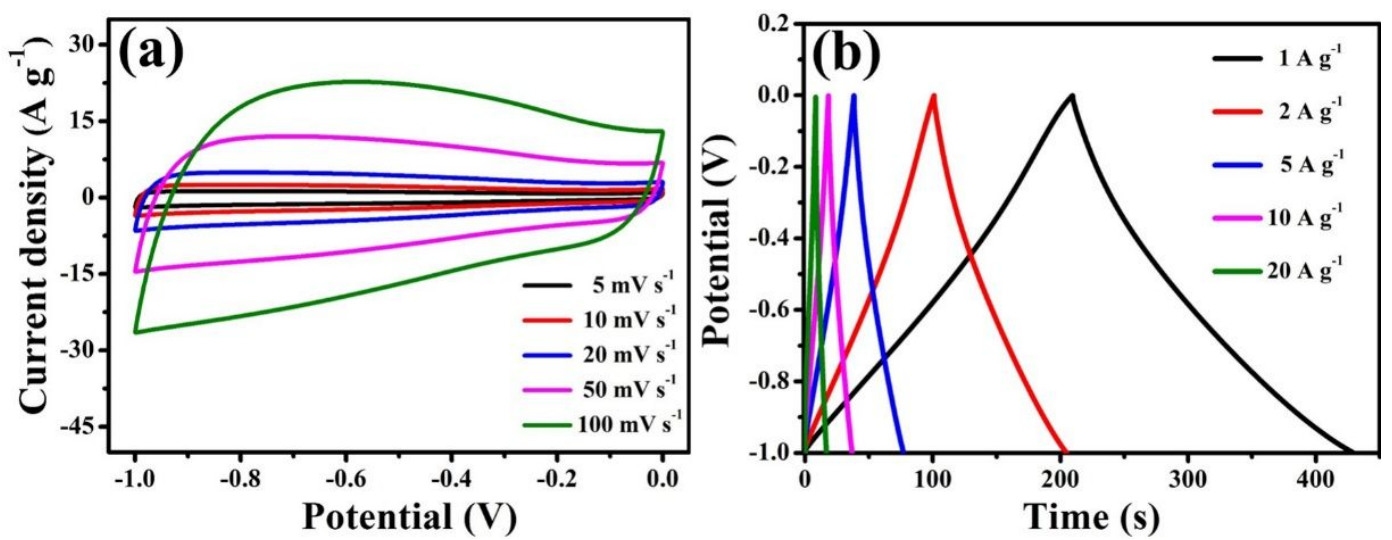

Figure S14. (a) CV curves and (b) GCD curves of AC electrode. 


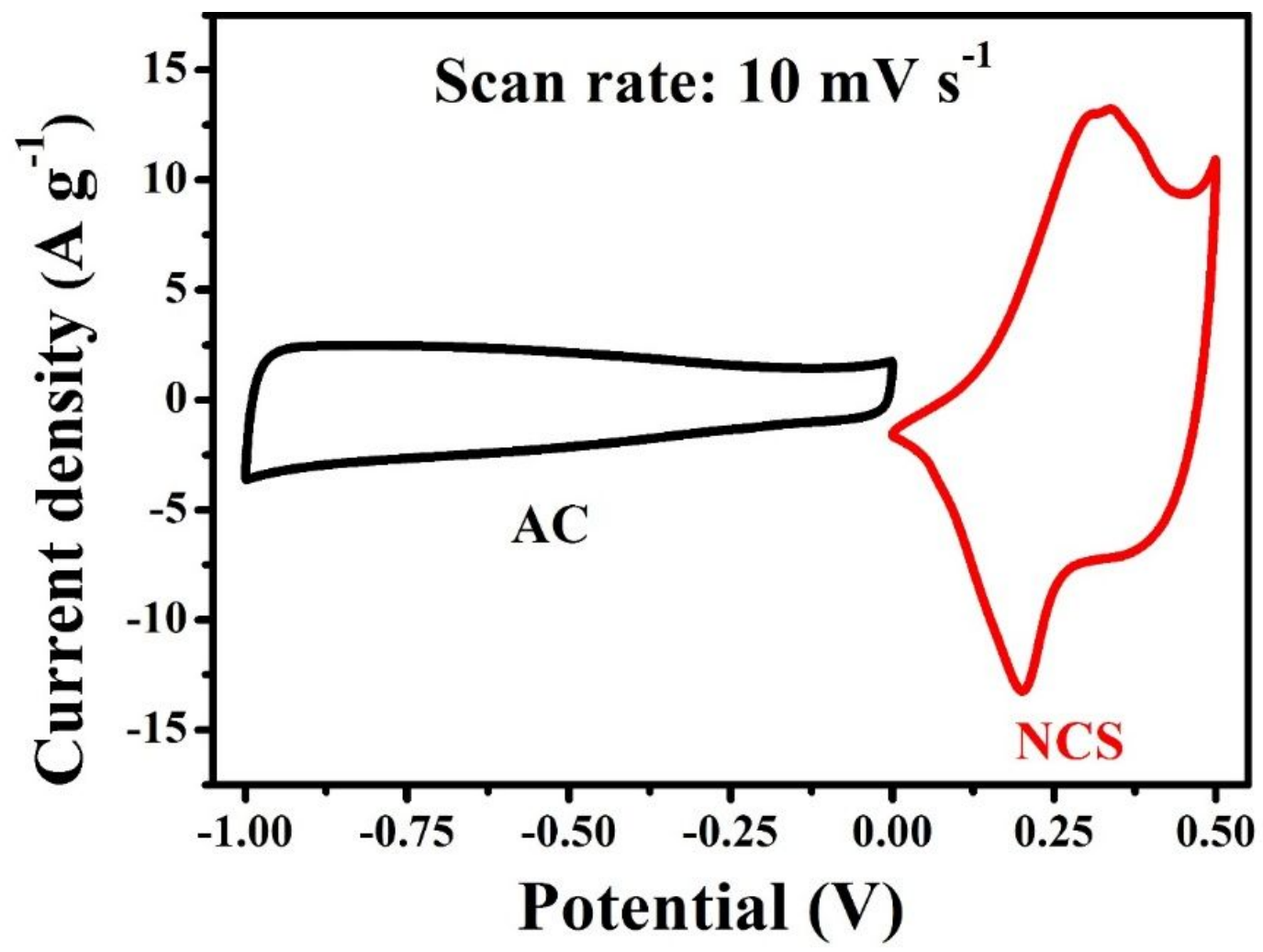

Figure S15. CV curves of AC and NCS electrode in $2 \mathrm{M} \mathrm{KOH}$ aqueous electrolyte. 

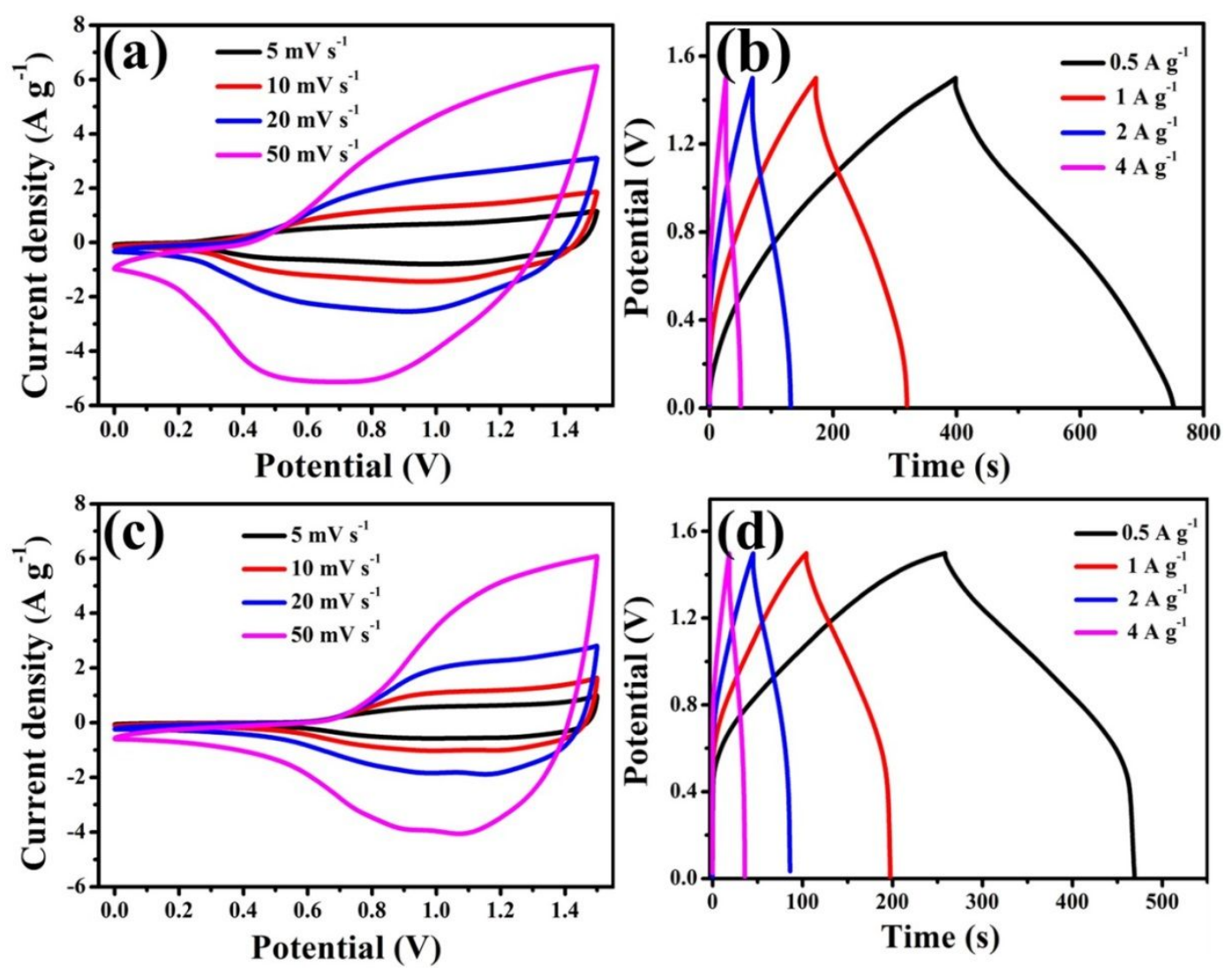

Figure S16. (a) CV curves and (b) GCD curves of NCS/NGF\\AC ASC. (c) CV curves and (d) GCD curves of NCS $\backslash$ AC ASC.

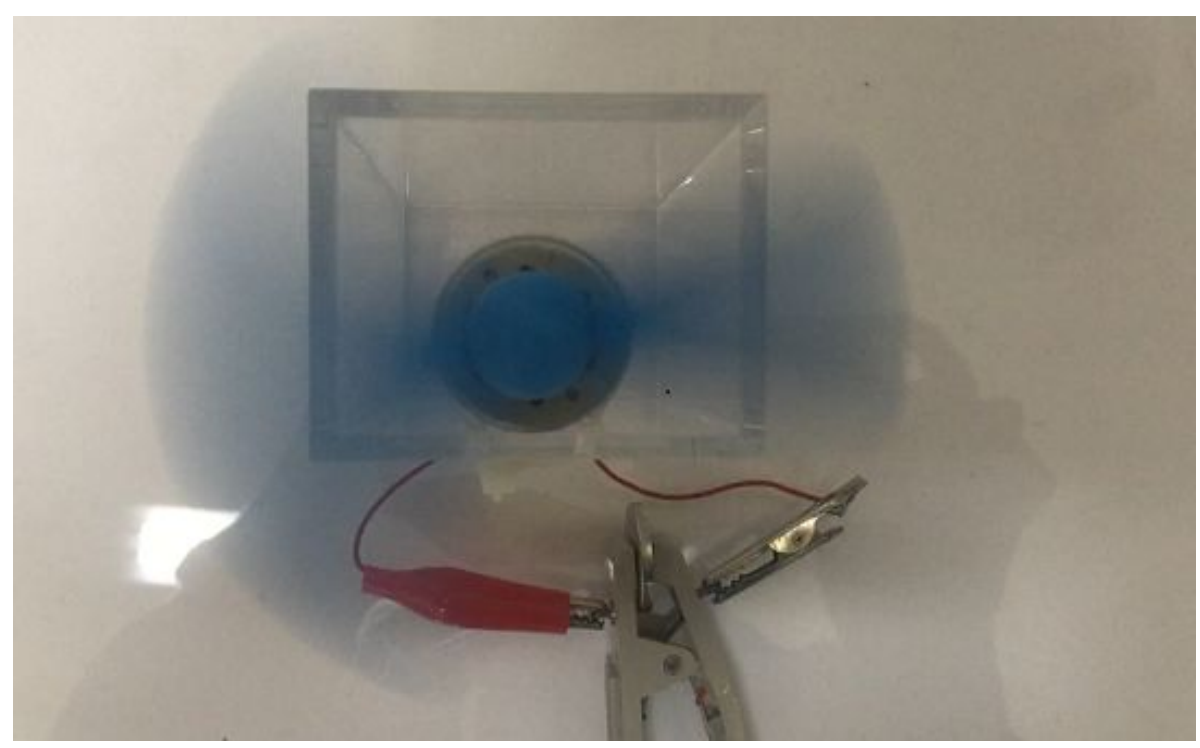

Figure S17. Small fan motored by a single NCS/NGF\\AC ASC device. 


\section{References}

(1) Fan, Y.-M.; Liu, Y.; Liu, X.; Liu, Y.; Fan, L.-Z. Hierarchical porous $\mathrm{NiCo}_{2} \mathrm{~S}_{4}-\mathrm{rGO}$ composites for high-performance supercapacitors. Electrochim. Acta 2017, 249, 1-8, DOI: 10.1016/j.electacta.2017.07.175.

(2) Hou, L. R.; Shi, Y. Y.; Zhu, S. Q.; Rehan, M.; Pang, G.; Zhang, X. G.; Yuan, C. Z. Hollow mesoporous hetero- $\mathrm{NiCo}_{2} \mathrm{~S}_{4} / \mathrm{Co}_{9} \mathrm{~S}_{8}$ submicro-spindles: unusual formation and excellent pseudocapacitance towards hybrid supercapacitors. J. Mater. Chem. A 2017, 5 (1), 133-144, DOI: 10.1039/c6ta05788h.

(3) Wan, H.; Jiang, J.; Yu, J.; Xu, K.; Miao, L.; Zhang, L.; Chen, H.; Ruan, Y. $\mathrm{NiCo}_{2} \mathrm{~S}_{4}$ porous nanotubes synthesis via sacrificial templates: high-performance electrode materials of supercapacitors. CrystEngComm 2013, 15 (38), 7649-7651, DOI: $10.1039 / \mathrm{c} 3 \mathrm{ce} 41243 \mathrm{a}$.

(4) Wu, Z.; Pu, X.; Ji, X.; Zhu, Y.; Jing, M.; Chen, Q.; Jiao, F. High Energy Density Asymmetric Supercapacitors From Mesoporous $\mathrm{NiCo}_{2} \mathrm{~S}_{4}$ Nanosheets. Electrochim. Acta 2015, 174, 238-245, DOI: 10.1016/j.electacta.2015.06.011.

(5) Zheng, Y.; Wang, X.; Zhao, W.; Cao, X.; Liu, J. Phytic acid-assisted synthesis of ultrafine $\mathrm{NiCo}_{2} \mathrm{~S}_{4}$ nanoparticles immobilized on reduced graphene oxide as highperformance electrode for hybrid supercapacitors. Chem. Eng. J. 2018, 333, 603-612, DOI: 10.1016/j.cej.2017.10.008.

(6) Zhu, Y.; Wu, Z.; Jing, M.; Yang, X.; Song, W.; Ji, X. Mesoporous $\mathrm{NiCo}_{2} \mathrm{~S}_{4}$ nanoparticles as high-performance electrode materials for supercapacitors. J. Power Sources 2015, 273, 584-590, DOI: 10.1016/j.jpowsour.2014.09.144. 
(7) Zheng, Y.; Xu, J.; Yang, X.; Zhang, Y.; Shang, Y.; Hu, X. Decoration $\mathrm{NiCo}_{2} \mathrm{~S}_{4}$ nanoflakes onto Ppy nanotubes as core-shell heterostructure material for highperformance asymmetric supercapacitor. Chem. Eng. J. 2018, 333, 111-121, DOI: 10.1016/j.cej.2017.09.155.

(8) Chen, H.; Jiang, J.; Zhang, L.; Wan, H.; Qi, T.; Xia, D. Highly conductive $\mathrm{NiCo}_{2} \mathrm{~S}_{4}$ urchin-like nanostructures for high-rate pseudocapacitors. Nanoscale 2013, 5 (19), 8879-8883, DOI: 10.1039/c3nr02958a.

(9) Guan, B. Y.; Yu, L.; Wang, X.; Song, S.; Lou, X. W. Formation of Onion-Like $\mathrm{NiCo}_{2} \mathrm{~S}_{4}$ Particles via Sequential Ion-Exchange for Hybrid Supercapacitors. Adv. Mater. 2017, 29 (6), 1605051, DOI: 10.1002/adma.201605051.

(10) Pu, J.; Cui, F.; Chu, S.; Wang, T.; Sheng, E.; Wang, Z. Preparation and Electrochemical Characterization of Hollow Hexagonal $\mathrm{NiCo}_{2} \mathrm{~S}_{4}$ Nanoplates as Pseudocapacitor Materials. ACS. Sustain. Chem. Eng. 2013, 2 (4), 809-815, DOI: $10.1021 / \mathrm{sc} 400472 \mathrm{z}$

(11) Li, G.; Xu, C. Hydrothermal synthesis of $3 \mathrm{D} \mathrm{Ni}_{\mathrm{x}} \mathrm{Co}_{1-\mathrm{x}} \mathrm{S}_{2}$ particles/graphene composite hydrogels for high performance supercapacitors. Carbon 2015, 90, 44-52, DOI: 10.1016/j.carbon.2015.03.066.

(12) Liu, T.; Jiang, C.; Cheng, B.; You, W.; Yu, J. Hierarchical NiS/N-doped carbon composite hollow spheres with excellent supercapacitor performance. J. Mater. Chem. $A$ 2017, 5 (40), 21257-21265, DOI: $10.1039 / \mathrm{c} 7 \mathrm{ta} 06149 \mathrm{~h}$.

(13) Wang, T.; Le, Q.; Zhang, G.; Zhu, S.; Guan, B.; Zhang, J.; Xing, S.; Zhang, Y. Facile preparation and sulfidation analysis for activated multiporous carbon $@ \mathrm{NiCo}_{2} \mathrm{~S}_{4}$ 
nanostructure with enhanced supercapacitive properties. Electrochim. Acta 2016, 211, 627-635, DOI: 10.1016/j.electacta.2016.06.082.

(14) Liang, X.; Nie, K.; Ding, X.; Dang, L.; Sun, J.; Shi, F.; Xu, H.; Jiang, R.; He, X.; Liu, Z.; Lei, Z. Highly Compressible Carbon Sponge Supercapacitor Electrode with Enhanced Performance by Growing Nickel-Cobalt Sulfide Nanosheets. ACS Appl. Mater. Interfaces 2018, 10 (12), 10087-10095, DOI: 10.1021/acsami.7b19043. 